\title{
(2) OPEN ACCESS \\ Assessment and management of peripheral arterial disease: what every cardiologist should know
}

\author{
Bao Tran
}

Correspondence to

Dr Bao Tran, Cardiology, St Mary's Medical Center, San Francisco, California, USA: Bao.Tran3@CommonSpirit.org

Check for updates

(c) Author(s) (or their employer(s)) 2021. Re-use permitted under CC BY-NC. No commercial re-use. See rights and permissions. Published by BMJ.

To cite: Tran B. Heart Epub ahead of print:

[please include Day Month Year]. doi:10.1136/

heartjnl-2019-316164

\section{INTRODUCTION}

Peripheral artery disease (PAD) typically refers to atherosclerotic narrowing and/or occlusion of all arterial disease other than coronary arteries and the aorta (carotid and vertebral arteries, coeliac and mesenteric arteries, renal arteries and upper and lower limb arteries) (figure 1). As a manifestation of systemic atherosclerosis, PAD is associated with greatly increased cardiovascular morbidity and mortality and impairment in quality of life. Because of the frequent overlap between ischaemic heart disease and PAD, cardiologists are in a unique position to screen, diagnose and treat PAD. The body of clinical evidence in patients with PAD is not as robust as the evidence in patients with heart disease, however the treatment goals are similar for both coronary artery disease $(\mathrm{CAD})$ and PAD: to prevent ischaemic outcomes with lifestyle changes and medical therapy and to weigh the risks and benefits of revascularisation (Table 1). This article reviews the basic evaluation and management of the most common types of PAD from a cardiologist's perspective.

\section{EPIDEMIOLOGY OF PAD}

The epidemiology for different patterns of PAD (carotid artery disease, upper extremity artery disease, mesenteric artery disease, renal artery disease and lower extremity artery disease) are varied depending on population of interest. They all share common risk factors for atherosclerosis, such as smoking, hypertension, dyslipidaemia, diabetes and autoimmune/inflammatory conditions such as systemic lupus erythematosus and rheumatoid arthritis. All patients with PAD should be screened for these risk factors.

Isolated $\mathrm{PAD}$ is an independent risk factor for cardiovascular event, and patients with vascular disease in multiple vascular beds carry the greatest risk for cardiovascular morbidity and mortality. However, once diagnosis of PAD is established in one vascular bed, there is no benefit in screening for asymptomatic atherosclerosis in other arterial beds as it would unlikely lead to change in management. In patients with significant $\mathrm{CAD}$, proactive ultrasound screening for PAD was not shown to be beneficial over routine medical therapy. ${ }^{1}$ Even in patients planned for coronary artery bypass grafting (CABG) who often get screening carotid ultrasound, there is no clear evidence supporting prophylactic carotid revascularisation in the absence of neurological symptoms.

\section{DIAGNOSIS AND ASSESSMENT OF PAD}

In addition to the standard medical history and assessment for cardiac patients, patient should be asked about neurological symptoms, exertional arm pain, exertional dizziness or vertigo to screen

\section{Learning objectives}

- To be familiar with tools in the diagnosis of peripheral artery disease (PAD), including the ankle brachial index and various imaging modalities.

- To implement general treatment modalities, including lifestyle modification, supervised exercise training programme and medical therapy for patients with PAD.

- To weigh the risks and benefits of invasive (interventional and surgical) management in PAD.

for carotid, vertebral and upper extremity PAD. Abdominal pain if related to eating can suggest mesenteric disease. Patients should also be screened for claudication and poorly healing wounds of the extremities. Early recognition of ischaemic ulcer can help prevent tissue loss and amputation. All vascular beds should be palpated for pulses and auscultated for bruit. Blood pressure measurement of both arms should be done-an interarm difference of $15 \mathrm{~mm} \mathrm{Hg}$ should raise the question of subclavian artery disease.

\section{ANKLE BRACHIAL INDEX}

The ankle brachial index (ABI) is a simple bedside tool to diagnose lower extremity PAD. The ABI of each leg is the highest ankle systolic blood pressure (SBP) (obtained by blood pressure cuff above the ankle and Doppler of both the dorsalis pedis and posterior tibial arteries) divided by the highest arm SBP. A low ABI $(<0.9)$ is indicative of obstructive disease, and a very elevated ABI $(>1.4)$ represents arterial stiffening from calcification. In patients with claudication symptoms, exercise treadmill ABI or pedal plantarflexion (toe raises) $\mathrm{ABI}$ increase the sensitivity of the test.

In patients who are elderly or with diabetes or chronic kidney disease (CKD), $\mathrm{ABI}$ is often elevated due to medial calcification. If there is suspicion that $\mathrm{ABI}$ is artificially elevated, toe brachial index (TBI) should be measured because the digital arteries are rarely non-compressible. A TBI of $<0.7$ is generally considered to be abnormal. Due to high likelihood of falsely elevated ABI in patients with chronic limb-threatening ischaemia (CLTI), TBI should always be obtained in patients with lower extremity wounds. Other measurements of perfusion, such as transcutaneous oxygen pressure and skin perfusion pressure, can help guide revascularisation in patients with CLTI. 


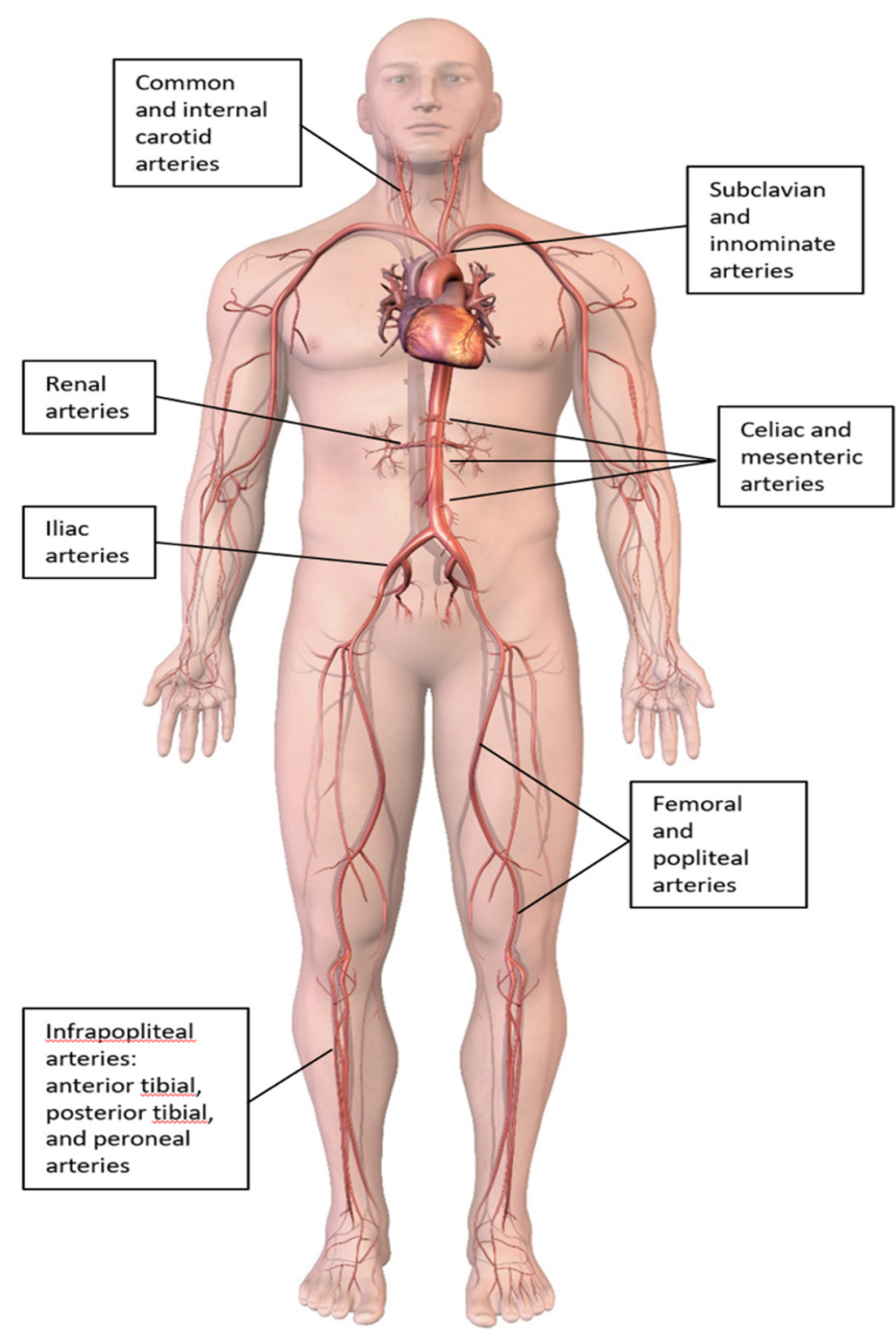

Figure 1 Common sites of disease in patients with peripheral artery disease.

\section{IMAGING}

Duplex ultrasound (DUS) is an excellent modality for both screening and diagnosis of PAD. It provides location and extent of disease as well as haemodynamics information. For evaluation of carotid disease or lower extremity disease, DUS often provides sufficient data. DUS evaluation of other arterial vascular beds depends heavily on operator experience and patient's body habitus.

Multidetector CT angiography (CTA) is often the next step in the diagnostic algorithm. It provides a high-resolution three-dimensional roadmap to confirm diagnosis and can help determine the optimal interventional strategy. The downside compared with ultrasound is cost, lack of functional data, radiation exposure and the need for iodinated contrast agents. CT imaging is more accurate than ultrasound for vertebral artery disease, subclavian artery disease, mesenteric artery disease, aorto-iliac disease and renal artery disease.

The role of magnetic resonance angiography (MRA) is more limited in PAD. Compared with CTA, motion artefact is more frequent in MRA and can often degrade image quality of smaller vessels. MRA also underestimates vascular calcification and cannot visualise the lumen of most endovascular stents. MRA is useful in patients with moderate CKD to avoid iodinated contrast, however in patients with advanced CKD and end-stage renal disease, there remains concerns for nephrogenic systemic fibrosis.

Invasive angiogram with digital subtraction angiography is the diagnostic gold standard and is typically reserved for patients who also need concomitant endovascular revascularisation. Carbon dioxide is an alternative contrast agent in the cath lab that permits endovascular procedures to be done safely in patients with advanced kidney disease. ${ }^{2}$

\section{GENERAL TREATMENT MODALITIES}

Tobacco use is one of the strongest risk factors for development, progression and recurrence of PAD after revascularisation. The benefits of smoking cessation rivals that of many pharmacological therapies. ${ }^{3}$ Similar to patients with CAD, patients with PAD should also receive specific instructions on lifestyle modification, such as structured exercise, dietary intervention, and weight management.

\section{ANTIPLATELET AND ANTITHROMBOTIC THERAPY}

While aspirin is generally recommended for all patients with symptomatic PAD, the ESC guidelines give a stronger level of recommendation to clopidogrel over aspirin based on CAPRIE (clopidogrel versus aspirin in patients at risk of ischaemic events) trial. ${ }^{4}$ Dual antiplatelet therapy (DAPT) is

Table 1 Parallel concepts in care in patients with CAD and PAD

\begin{tabular}{|c|c|c|}
\hline CAD & PAD & Management \\
\hline Guideline-directed medical therapy & Optimal medical therapy & Antithrombotic, lipid-lowering, antihypertensive and glycaemic control agents \\
\hline Lifestyle management & Lifestyle management & Smoking cessation, dietary intervention, weight management, exercise \\
\hline Stable angina & Claudication & $\begin{array}{l}\text { Conservative symptom management with revascularisation reserved for } \\
\text { significant symptoms }\end{array}$ \\
\hline Acute coronary syndrome & $\begin{array}{l}\text { Chronic limb-threating ischaemia and chronic } \\
\text { mesenteric ischaemia }\end{array}$ & Revascularisation generally preferred over medical therapy alone \\
\hline
\end{tabular}


often used in patients postrevascularisation for at least 1 month, however there are no randomised data supporting specific agent or duration of therapy. Warfarin is sometimes used after bypass graft, however evidence has been mixed comparing efficacy of warfarin against aspirin alone or DAPT. Ticagrelor is a more potent $\mathrm{P} 2 \mathrm{Y} 12$ receptor antagonist commonly used in patients with acute coronary syndrome. But when randomised against clopidogrel in patients with symptomatic PAD, ticagrelor failed to show significant clinical benefits. ${ }^{5}$ Vorapaxar, a thrombin receptor antagonist, has a weak class IIb recommendation in the American College of Cardiology (ACC) guidelines based on a trial showing reduced ischaemic limb events. ${ }^{6}$ However, vorapaxar has not gained popularity likely due to increased bleeding risk without significant reduction of major adverse cardiovascular events (MACE).

For patients with claudication, cilostazol is recommended by ACC guidelines (but not by European Society of Cardiology (ESC)/European Society for Vascular Surgery (ESVS) guidelines) for symptom reduction. In our experience, cilostazol only provides modest efficacy with a relatively high discontinuation rate due to side effects. Pentoxifylline is an older medication for claudication and is no longer recommended by current major guidelines.

The next version of the guidelines will likely include recommendations about low-dose rivaroxaban, an oral direct factor Xa inhibitor. COMPASS (cardiovascular outcomes for people using anticoagulation strategies) trial randomised patients with lower extremity peripheral artery disease or significant carotid artery disease to lowest-dose rivaroxaban ( $2.5 \mathrm{mg}$ twice a day) in addition to aspirin, lower-dose rivaroxaban $(5 \mathrm{mg}$ twice a day) alone or aspirin alone. ${ }^{7}$ Combination of lowest-dose rivaroxaban and aspirin had the lowest composite end point of cardiovascular death, myocardial infarction or stroke as well as major adverse limb events. In the VOYAGER-PAD (vascular outcomes study of ASA (acetylsalicylic acid) along with rivaroxaban in endovascular or surgical limb revascularization for PAD (peripheral artery disease)) trial, patients with PAD who are undergoing either surgical or endovascular revascularisation were randomised to low-dose rivaroxaban $(2.5 \mathrm{mg}$ twice a day) in addition to aspirin or aspirin alone. ${ }^{8}$ Addition of low-dose rivaroxaban to aspirin again was shown to reduce ischaemic outcomes. In both trials, reduction in ischaemic events come with a small increased risk of non-fatal bleeding in the combination group when compared with aspirin alone.

\section{ANTIHYPERTENSIVE THERAPY}

There is overwhelming evidence supporting treatment hypertension to reduce cardiovascular events, and recent guidelines have pushed for tighter blood pressure control. However, there have been lingering concern that in patients with obstructive PAD, aggressive antihypertensive therapy may reduce limb perfusion, despite no large study having ever shown this. Treatment of blood pressure should follow established guidelines, and the choice of specific agents should be dictated by patient's comorbidities. Of note, ACE inhibitor (ACEI) has been shown to reduce all-cause mortality in both symptomatic and subclinical patients with PAD in the HOPE (heart outcomes prevention evaluation) trial. ${ }^{9}$

\section{LIPID-LOWERING THERAPY}

Statins have been shown to consistently reduce risk of stroke and heart attack in patients with ischaemic heart disease by reducing cholesterol as well as through other cholesterol-independent pleiotropic effects. In patients with PAD, simvastatin significantly reduced MACE in the Heart Protection Study. ${ }^{10}$ Preprotein convertase subtisilin/kexin type 9 (PCSK-9) antibodies have also been shown to reduce ischaemic events in patients with CAD who do not meet low-density lipoprotein goals despite maximally tolerated statin. The benefits of PCKS-9 extend to patients with isolated PAD. In the FOURIER (further cardiovascular outcomes research with PCSK9 inhibition in subjects with elevated risk) randomised trial, evolocumab significantly reduced the risk of MACE and major adverse limb events (defined as acute limb ischaemia (ALI), urgent limb revascularisation and amputation) in patients with isolated symptomatic PAD. ${ }^{11}$ In the ODYSSEY OUTCOMES (evaluation of cardiovascular outcomes after an acute coronary syndrome during treatment with alirocumab) randomised trial, alirocumab showed the highest absolute risk reduction in patients with polyvascular disease in three beds (coronary, peripheral and cerebrovascular) compared with patients with isolated $\mathrm{CAD}{ }^{12}$ Another emerging therapy for ischaemic heart disease, icosapent ethyl, will be evaluated in patients with PAD in the near future.

\section{CAROTID ARTERY DISEASE}

Approximately $10 \%-20 \%$ of thromboembolic stroke is thought to be from significant extracranial carotid artery disease. Generally, significant stenosis refers to a $>50 \%$ stenosis estimated using the North American Symptomatic Carotid Endarterectomy Trial method. ${ }^{13}$ In clinical trials, patients are often stratified to $50 \%-69 \%$ stenosis and 70\%-99\% stenosis. Revascularisation is not indicated for a near-occluded or occluded carotid artery. A carotid stenosis is defined as 'symptomatic' if there is ischaemic symptoms within preceding 6 months. Abnormalities on brain imaging studies without symptoms are generally not considered 'symptomatic'.

Early revascularisation is well-established as the standard of care for patients with symptomatic carotid artery stenosis (figure 2). Multiple randomised clinical trials (RCTs) have consistently found carotid endarterectomy (CEA) to be superior to medical therapy alone in this population. ${ }^{14}$ Clinical features associated with higher benefits from CEA are more severe carotid lesions (70\%-99\%), 


\section{Carotid Artery \\ Disease in \\ Symptomatic \\ Patients*}
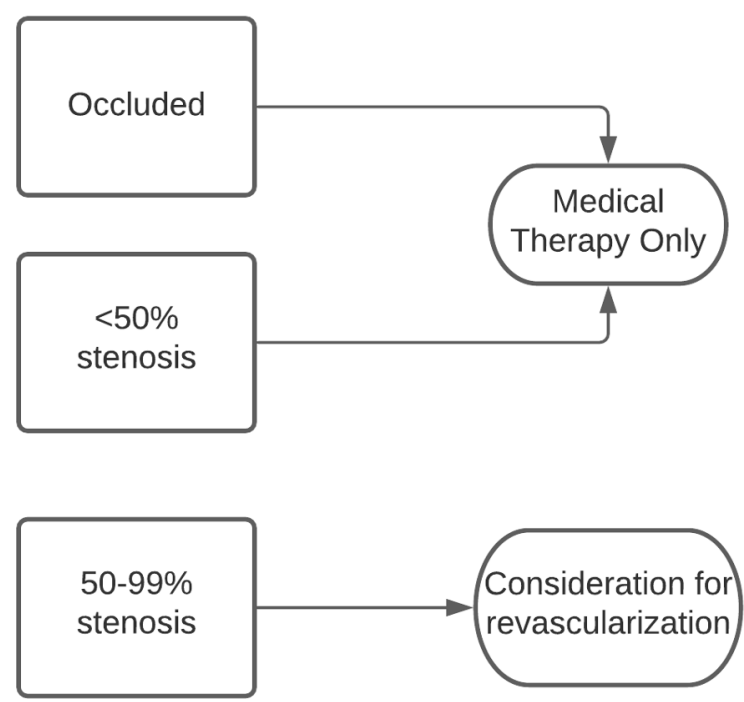

Figure 2 Diagnostic algorithm for patients with symptomatic carotid artery disease. *Symptoms of stroke/transient ischaemic attack within 6 months.

male sex, older age (>75 years) and hemispheric and cortical strokes (as opposed to retinal or lacunar strokes). The benefits of CEA are greatest within 14 days of the ischaemic event and decline as time passes.

The management of asymptomatic carotid disease is more controversial. Early trials found better outcomes with CEA compared with medical therapy for significant carotid disease in asymptomatic patients. However, medical therapy has evolved greatly since the 1990s and early 2000s when there were little statin use, no antithrombotic option other than aspirin and no specific goal for treating blood pressure and diabetes. Lacking newer data, the ESC/ ESVS guideline only recommends revascularisation in asymptomatic patients with 60\%-99\% who have additional risk factors, such as a history of

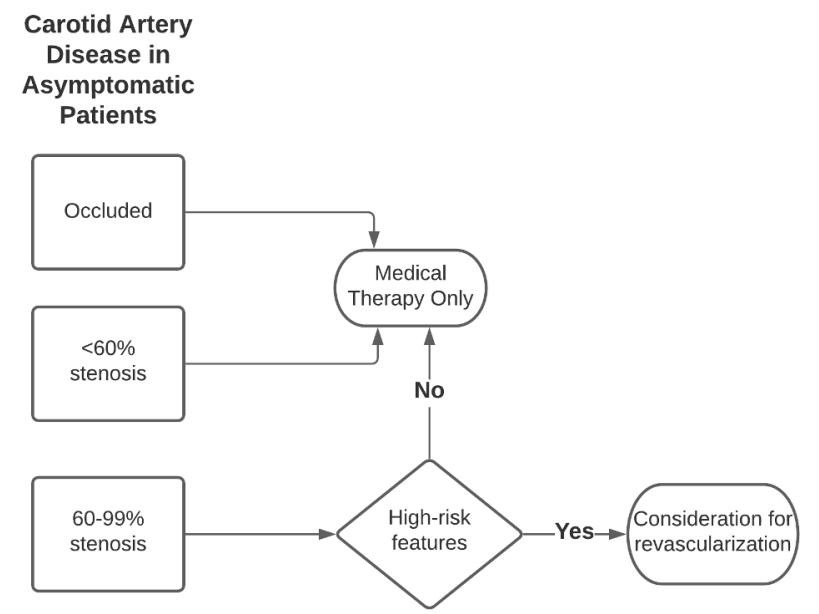

Figure 3 Diagnostic algorithm for patients with asymptomatic carotid artery disease. contralateral transient ischaemic attack/stroke, ipsilateral silent infarction or anatomically high-risk findings on imaging (lipid-rich core or intraplaque haemorrhage on MRA, large echolucent and hypoechogenic plaque on ultrasound or rapid stenosis progression) (Figure 3). The ongoing CREST-2 (carotid revascularization for primary prevention of stroke) trial will help determine whether CEA, carotid artery stenting (CAS) or modern medical therapy is the best strategy for patients with asymptomatic significant carotid stenosis. ${ }^{15}$

Although the revascularisation data are strongest for CEA, there are multiple trials comparing CEA with CAS. Overall, CAS is associated with higher risk of stroke and mortality but lower risk of perioperative MI when compared with CEA. ${ }^{16}$ Thus, CEA remains the default option except in patients considered to be at high surgical risk. High surgical risk may include significant cardiopulmonary disease, hostile neck (previous neck surgery or radiation therapy), contralateral internal carotid artery occlusion, contralateral recurrent laryngeal nerve palsy and advanced age. Additionally, there are concerns that the real-world stroke rates of CAS in low-volume centres may be higher than those reported in clinical trials. Thus, for an asymptomatic patients to benefit from prophylactic procedure, CAS should be performed in an experienced centre where the risk of periprocedural stroke and death must be $<3 \%$. If the patient is symptomatic, CAS may be considered if the expected periprocedural stroke and death is $<6 \%$.

Transcarotid artery revascularisation (TCAR) is a novel hybrid revascularisation approach that is gaining popularity. ${ }^{17}$ TCAR combines surgical elements (direct surgical cutdown and clamping of the common carotid artery) with endovascular elements (deployment of a stent) along with additional embolic protection with a flow reversal system. Due to a perceived lower perioperative mortality compared with CEA and lower periprocedure stroke compared with CAS, the number of TCAR procedures being performed has grown rapidly in the past few years at the expense of carotid stenting. The benefits of TCAR however still need to be confirmed by an RCT.

Vertebral artery disease accounts for up to $20 \%$ of posterior circulation ischaemic events. A preplanned pooled individual patient analysis from three RCTs comparing stenting with medical therapy alone in patients with symptomatic vertebral artery stenosis showed no benefit with stenting and significant risk of periprocedural stroke. ${ }^{18}$ Open surgery (transposition or bypass) is not typically done except in specialised centres. Thus, revascularisation of vertebral artery disease should be reserved only for exceptional cases.

\section{UPPER EXTREMITY PAD}

Atherosclerotic upper extremity PAD occurs most commonly in left subclavian artery, followed by right subclavian artery and then innominate artery. It should be suspected if there is $>15 \mathrm{~mm} \mathrm{Hg} \mathrm{SBP}$ 


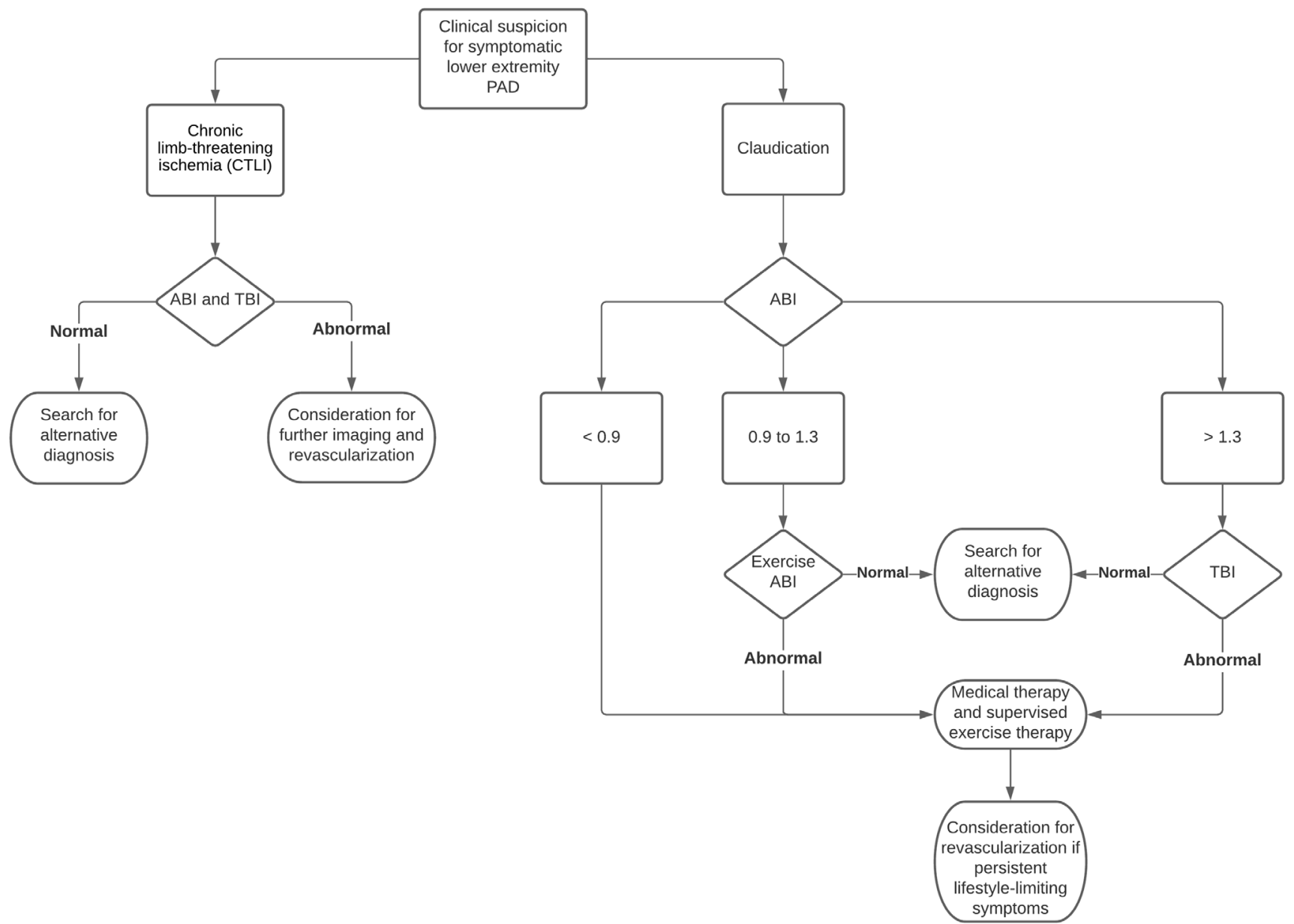

Figure 4 Diagnostic algorithm for patients with lower extremity artery disease. $A B I$, ankle brachial index; PAD, peripheral artery disease; $T B I$, toe brachial index.

difference between each arm, although patients are typically only symptomatic with more severe stenosis causing higher blood pressure differences. Symptoms may include upper extremity claudication, vertebral basilar steal, coronary steal in patients with internal mammary artery coronary bypass grafts or dialysis access dysfunction. Revascularisation is indicated only for symptomatic disease or in cases of planned CABG using internal mammary artery. Although there are no data directly comparing surgical and endovascular treatment, endovascular treatment with balloon-expandable stent is often preferred first-line therapy due to high success rate and high long-term patency rate. ${ }^{19}$

\section{MESENTERIC ARTERY DISEASE}

Acute or chronic mesenteric ischaemia is uncommon but carries high mortality rates if not treated promptly. The classic symptoms of chronic mesenteric artery disease are food aversion from postprandial abdominal pain, weight loss and diarrhoea or constipation. Due to robust collateral circulation patients typically only develop symptoms when there is severe disease in two out of the three main arterial systems: coeliac trunk, superior mesenteric artery and inferior mesenteric artery. Revascularisation is indicated for all symptomatic patients with chronic mesenteric ischaemia. As these patients tend to be older with multiple comorbidities, endovascular approach with primary stenting is typically the first option to minimise perioperative complications. ${ }^{20}$ If patient is a lower risk surgical candidate, surgical repair offers more symptom relief and higher primary patency but at the expense of slower recovery and longer hospital stays compared with endovascular repair.

Acute mesenteric ischaemia occurs most commonly in the superior mesenteric artery from an embolic source, although thrombotic occlusion from atherosclerotic disease can sometimes be seen as well. Mortality is very high if diagnosis or treatment is delayed. The classic presentation is acute severe abdominal pain that is out of proportion to the physical exam (soft abdomen without rebound or guarding). D-dimer is highly sensitive for acute mesenteric ischaemia, but specificity is poor. $^{21}$ There is no RCT comparing open surgery with endovascular therapy, so treatment often depends on local expertise. An advantage of open surgery is the ability to concomitantly inspect the bowel for gangrene, necrosis and perforation. At the time 
of laparotomy, hybrid revascularisation can also be performed with direct puncture of the mesenteric artery followed by stenting.

\section{RENAL ARTERY DISEASE}

Atherosclerotic renal artery stenosis (RAS) is the most common cause of renovascular hypertension in adults, however in younger women fibromuscular dysplasia (FMD) is the most common cause of renovascular hypertension. Luminal obstruction and hypoperfusion of the kidney activates the renin-angiotensin-aldosterone system and leads to systemic hypertension. All patients with significant atherosclerotic RAS should be managed with blood pressure control, statin and antiplatelet therapy. Patients with bilateral RAS can still safely be treated with ACEIs or angiotensin receptor blockers, provided that they have close follow-up of renal function.

Three major RCTs (STAR (Stent placement and blood pressure and lipid-lowering for the prevention of progression of renal dysfunction caused by atherosclerotic ostial stenosis of the renal artery), ASTRAL (angioplasty and stenting for renal artery lesions) and CORAL (cardiovascular outcomes in renal atherosclerotic lesions) have not found benefit for revascularisation over medical therapy, although these trials have been criticised for flaws in trial design and inclusion of some patients with only moderate disease. ${ }^{22}$ Still, due to lack of supporting evidence, renal revascularisation should be reserved for selected patients with FMD, recurrent congestive heart failure and flash pulmonary oedema, refractory hypertension despite adequate medical therapy (at least three antihypertensive agents including a diuretic agent) or progressive renal failure with renal ischaemia. Because of the associated morbidity and mortality of open surgery, endovascular treatment is the preferred technique for patients who need revascularisation. If a lesion is not obviously severe at the time angiography, confirmation of haemodynamic significance can be performed with measuring translesional systolic pressure gradient or renal fractional flow reserve. Before planned revascularisation, renal resistive index obtained by ultrasound can provide additional information on viability of the kidney and predicted response to revascularisation.

\section{LOWER EXTREMITY PAD—CLAUDICATION}

Patients with claudication tend to have stable symptoms with only a very small percentage progressing to CLTI. In addition to medical therapy, supervised exercise therapy is a cornerstone of treatment for claudication. Supervised exercise has been shown to improve walking performance compared with stenting in the CLEVER (claudication: exercise versus endoluminal revascularization) trial. ${ }^{23}$ But revascularisation combined with exercise offers the greatest improvement in walking distance and quality of life score. ${ }^{24}$ Unfortunately, supervised exercise therapy for claudication is not widely available. Revascularisation should be considered for lifestyle-limiting claudication after failure of an adequate trial of exercise therapy. Whether surgical revascularisation or endovascular revascularisation is chosen depends on anatomic complexity and local expertise. Endovascular techniques and equipment has evolved rapidly to increase success rate of procedure as well as allow procedure to be done through radial approach or transpedal approach. With aorto-iliac disease, endovascular approach is generally accepted as first-line therapy because of high patency rate of stents. For common femoral artery disease, surgical endarterectomy is the standard of care because it is highly effective with low perioperative risk. For femoropopliteal disease, decision between surgical bypass and endovascular therapy depends on anatomic complexity of occlusion, presence of venous conduit and patient's surgical risk. If surgical bypass is decided, an autologous vein as a bypass conduit has much higher long-term patency compared with prosthetic grafts. If endovascular approach is chosen first, landing zones for potential future bypass grafts should be preserved.

\section{LOWER EXTREMITY PAD-CHRONIC LIMB- THREATENING ISCHAEMIA}

CLTI is an advanced form of peripheral artery disease encompassing rest pain, lower limb ulceration or gangrene. It is associated with significant morbidity, mortality and healthcare resource utilisation. The term CLTI is the preferred term over the older nomenclature critical limb ischaemia (CLI). The burden of obstructive disease in patients with CLTI often can involve multiple levels (aortoiliac, fem-pop and tibial vessels) with complex and calcified occlusions. An active wound requires a high level of blood flow to heal adequately, so the primary goal of revascularisation is to achieve direct inline flow to the wound bed.

For patients with CLTI, the Fontaine and Rutherford classification systems have traditionally been used to stratify patients from asymptomatic to tissue loss. While they are easy to use at the bedside to classify risk of amputation, both scales are insufficient in determining optimal therapy. The Wound, Ischaemia and Foot Infection (WIFI) classification system, developed by the Society for Vascular Surgery, was designed to provide more granular data and to also function as a clinical decision-making tool. ${ }^{25}$ WIFI gives a composite score to predict amputation risk and determine benefit of revascularisation. Multiple other classification systems exist for diabetic foot ulcer, but WIFI appears to be the most robust and widely used system currently.

Revascularisation is the mainstay of therapy for CLTI, but it is still uncertain which patients and which anatomy benefit from an 'endovascular first' approach or 'surgical bypass first' approach. To help answer this question, BEST-CLI (best endovascular versus best surgical therapy in patients with critical limb ischemia) band BASIL-2 (bypass versus angioplasty in severe ischaemia of the leg) are two 
ongoing RCTs comparing endovascular revascularisation with surgical bypass in patients with CLTI. $^{26}{ }^{27}$ If there is significant tissue necrosis or infectious gangrene, amputation may be necessary to remove necrotic tissue. However, major amputation leads to a significant decline in functional status and is associated with dramatically reduced survival. ${ }^{28}$ Revascularisation should be attempted in all patients with CLI before amputation (Figure 4).

CLTI is a complex disease that lends itself well to a multidisciplinary approach. Similar to the Heart Team, experts for both endovascular technique and open surgical technique should be represented. The full spectrum of care for the patients with CLTI should also include podiatrists, wound care specialists, physical rehabilitation specialists, diabetes specialists, infectious disease specialists, orthotists and prosthetists. The team should have strong hospital and clinic presence to facilitate the transition between inpatient and outpatient. Given the high morbidity of CLTI, the implementation of such a vascular team would streamline patient care and have potential to significantly improve outcomes and reduce cost to the system.

\section{ACUTE LIMB ISCHAEMIA}

Patients with ALI is a special subset that requires urgent evaluation and treatment. Similar to acute mesenteric ischaemia, aetiology is typically an

\section{Key messages}

- Isolated peripheral artery disease (PAD) is an independent risk factor for cardiovascular event, and patients with vascular disease in multiple vascular beds carry the greatest risk for cardiovascular morbidity and mortality.

- The ankle brachial index ( $A B I)$ is a simple bedside tool to diagnose lower extremity PAD. Exercise treadmill $A B I$ increases the sensitivity of the test. Due to high likelihood of falsely elevated $A B I$ in patients with chronic limbthreating ischaemia (CLTI), toe brachial index should always be obtained in patients with lower extremity wounds.

- General treatment for PAD is similar to ischaemic heart disease and includes smoking cessation, lifestyle modification, antihypertensive therapy, and lipidlowering therapy. Recent evidence shows addition of low-dose rivaroxaban to aspirin has been shown to be effective in reducing ischaemic outcomes in patients with PAD.

- Patients with symptomatic signficant carotid artery disease should undergo revascularisation within 14 days of event. Carotid revascularisation for asymptomatic patients remain controversial and should be reserved only in specific higher risk scenarios.

- Acute or chronic mesenteric ischaemia is often misdiagnosed. Revascularisation is often necessary for symptomatic patients.

- Routine revascularisation of atherosclerotic renal artery stenosis (RAS) has not been shown to be beneficial compared with medical therapy alone. Revascularisation should be reserved for a highly selected group of patients.

- For patients with claudication, supervised exercise is the mainstay of therapy.

- Patients with CLTI have high rates of mortality, and prompt revascularisation is indicated as well as comprehensive care by a multidisciplinary group of providers.

- Although paclitaxel-coated devices offer longer primary patency than noncoated devices in femoropopliteal PAD, there remains lingering concern about its long-term safety. embolic source or thrombotic occlusion from underlying atherosclerotic disease. For patients with ALI, anticoagulation with heparin should immediately be started. Unlike ST-elevation myocardial infarction, there is no role for systemic thrombolysis in patients with ALI. The degree of sensory and motor deficit drives the urgency of revascularisation. If the limb is deemed to be viable, revascularisation options include surgical or percutaneous thrombectomy, catheter-based thrombolysis and ultrasoundaccelerated catheter-based thrombolysis. ${ }^{29}$ Older studies comparing open surgery with endovascular techniques do not include the use of modern thrombectomy systems that are very effective in thrombus removal. ${ }^{30}$ Regardless of revascularisation technique, surgeons should still be involved as patients with prolonged ischaemia can develop reperfusion injury and may require fasciotomies to prevent compartment syndrome.

\section{PACLITAXEL CONTROVERSY}

Drug-coated balloons (DCBs) and drug-eluting stents (DESs) coated with paclitaxel were gaining popularity in treatment of femoropopliteal PAD as they were shown to provide longer primary patency than non-coated devices. In 2018, a meta-analysis precipitated a firestorm in the vascular community when it showed excess mortality in patients receiving paclitaxel-coated devices for femoropopliteal interventions. ${ }^{31}$ The Medicines and Healthcare products Regulatory Agency and US Food and Drug Administration both issued warnings against the use of paclitaxel DCBs or DESs for patients. Many hospitals and physicians stopped using paclitaxel-coated devices, and important RCTs comparing various endovascular treatment strategies (such as BASIL-3 and SWEDEPAD) paused enrolment temporarily. However, many experts questioned the biological mechanism behind the increase in mortality, and subsequent datasets from ongoing clinical trials and real-world evidence have shown conflicting results. ${ }^{32}$ Currently, there remains a lack of consensus regarding the safety of paclitaxel-coated devices. The benefits of improved patency for individual patients must be weighed against the uncertain possibility of increased risk in mortality. This can be a difficult task when counselling a young patient with significant claudication. However, in patients with CLTI, paclitaxel-coated therapy is still the preferred therapy due to the high morbidity associated with recurrent ischaemia.

\section{CONCLUSION}

Despite sharing many common risk factors, the clinical presentation for various types PAD is diverse. All symptomatic patients with PAD should receive lifestyle modification, antithrombotic therapy, lipidlowering therapy and treatment for hypertension or diabetes. Decisions about revascularisation should be patient-centred and be discussed in a multidisciplinary fashion. Because of the broad spectrum of disease, there are still many gaps in evidence. RCTs often trail behind the rapid evolution in techniques 


\section{CME credits for Education in Heart}

Education in Heart articles are accredited for CME by various providers. To answer the accompanying multiple choice questions (MCQs) and obtain your credits, click on the 'Take the Test' link on the online version of the article. The MCQs are hosted on BMJ Learning. All users must complete a one-time registration on BMJ Learning and subsequently log in on every visit using their username and password to access modules and their CME record. Accreditation is only valid for 2 years from the date of publication. Printable CME certificates are available to users that achieve the minimum pass mark.

and technology. However, many parallels exist between caring for the 'cardiac' patient and caring for the 'vascular' patient. Cardiologists are in a unique position to provide optimal care for patients with PAD and drive future research and innovation.

Correction notice Since Online First publication, the second sentence of the 'ankle brachial index' section has been corrected to the following: 'The ABI of each leg is the highest arm systolic blood pressure (SBP) (obtained by blood pressure cuff above the ankle and Doppler of both the dorsalis pedis and posterior tibial arteries) divided by the highest ankle SBP'.

Twitter Bao Tran @BaoGTran

Funding The authors have not declared a specific grant for this research from any funding agency in the public, commercial or not-for-profit sectors.

Competing interests None declared.

Patient and public involvement Patients and/or the public were not involved in the design, or conduct, or reporting, or dissemination plans of this research.

Patient consent for publication Not required.

Provenance and peer review Commissioned; externally peer reviewed.

Author note References which include a * are considered to be key references.

Open access This is an open access article distributed in accordance with the Creative Commons Attribution Non Commercial (CC BY-NC 4.0) license, which permits others to distribute, remix, adapt, build upon this work non-commercially, and license their derivative works on different terms, provided the original work is properly cited, appropriate credit is given, any changes made indicated, and the use is non-commercial. See: http:// creativecommons.org/licenses/by-nc/4.0/.

\section{ORCID iD}

Bao Tran http://orcid.org/0000-0002-0980-7594

\section{REFERENCES}

1 Collet J-P, Cayla G, Ennezat P-V, et al. Systematic detection of polyvascular disease combined with aggressive secondary prevention in patients presenting with severe coronary artery disease: the randomized America study. Int J Cardiol 2018;254:36-42

2 Sharafuddin MJ, Marjan AE. Current status of carbon dioxide angiography. J Vasc Surg 2017;66:618-37.

3 Morris PB, Ference BA, Jahangir E, et al. Cardiovascular effects of exposure to cigarette smoke and electronic cigarettes: clinical perspectives from the prevention of cardiovascular disease section leadership Council and early career councils of the American College of cardiology. J Am Coll Cardiol 2015;66:1378-91.

4 CAPRIE Steering Committee. A randomised, blinded, trial of clopidogrel versus aspirin in patients at risk of ischaemic events (CAPRIE). CAPRIE Steering Committee. Lancet 1996;348:1329-39.
5 Hiatt WR, Fowkes FGR, Heizer G, et al. Ticagrelor versus clopidogrel in symptomatic peripheral artery disease. $N$ Engl J Med 2017;376:32-40.

*6 Kithcart AP, Beckman JA. ACC/AHA Versus ESC Guidelines for Diagnosis and Management of Peripheral Artery Disease: JACC Guideline Comparison. J Am Coll Cardiol 2018;72:2789-801.

*7 Anand SS, Bosch J, Eikelboom JW, et al. Rivaroxaban with or without aspirin in patients with stable peripheral or carotid artery disease: an international, randomised, double-blind, placebocontrolled trial. Lancet 2018;391:219-29.

8 Bonaca MP, Bauersachs RM, Anand SS, et al. Rivaroxaban in peripheral artery disease after revascularization. N Engl J Med 2020:382:1994-2004.

9 Östergren J, Sleight P, Dagenais G, et al. Impact of ramipril in patients with evidence of clinical or subclinical peripheral arteria disease. Eur Heart J 2004;25:17-24.

10 Heart Protection Study Collaborative Group. Randomized trial of the effects of cholesterol-lowering with simvastatin on peripheral vascular and other major vascular outcomes in 20,536 people with peripheral arterial disease and other high-risk conditions. $J$ Vasc Surg 2007; 45:645-54.

11 Bonaca MP, Nault P, Giugliano RP, et al. Low-Density lipoprotein cholesterol lowering with evolocumab and outcomes in patients with peripheral artery disease: insights from the Fourier trial (further cardiovascular outcomes research with PCSK9 inhibition in subjects with elevated risk). Circulation 2018;137:338-50.

12 Jukema JW, Szarek M, Zijlstra LE, et al. Alirocumab in Patients With Polyvascular Disease and Recent Acute Coronary Syndrome: ODYSSEY OUTCOMES Trial. J Am Coll Cardiol 2019:74:1167-76.

13 North American Symptomatic Carotid Endarterectomy Trial Collaborators, Barnett HJM, Taylor DW, et al. Beneficial effect of carotid endarterectomy in symptomatic patients with high-grade carotid stenosis. N Eng/ J Med 1991;325:445-53.

14 Rothwell PM, Eliasziw M, Gutnikov SA, et al. Analysis of pooled data from the randomised controlled trials of endarterectomy for symptomatic carotid stenosis. Lancet 2003:361:107-16.

15 Mott M, Koroshetz W, Wright CB. CREST-2: identifying the best method of stroke prevention for carotid artery stenosis: National Institute of neurological disorders and stroke organizational update. Stroke 2017;48:e130-1.

16 Economopoulos KP, Sergentanis TN, Tsivgoulis G, et al. Carotid artery stenting versus carotid endarterectomy: a comprehensive meta-analysis of short-term and long-term outcomes. Stroke 2011;42:687-92.

17 Kwolek CJ, Jaff MR, Leal Jl, et al. Results of the ROADSTER multicenter trial of transcarotid stenting with dynamic flow reversal. J Vasc Surg 2015;62:1227-34.

18 Markus HS, Harshfield EL, Compter A, et al. Stenting for symptomatic vertebral artery stenosis: a preplanned pooled individual patient data analysis. Lancet Neurol 2019;18:666-73

19 Saha T, Naqvi SY, Ayah OA, et al. Subclavian artery disease: diagnosis and therapy. Am J Med 2017;130:409-16.

20 Clair DG, Beach JM. Mesenteric ischemia. N Eng/ J Med 2016;374:959-68

21 Cudnik MT, Darbha S, Jones J, et al. The diagnosis of acute mesenteric ischemia: a systematic review and meta-analysis. Acad Emerg Med 2013;20:1087-100.

22 Prince M, Tafur JD, White CJ. When and how should we revascularize patients with atherosclerotic renal artery stenosis? JACC Cardiovasc Interv 2019;12:505-17.

23 Murphy TP, Cutlip DE, Regensteiner JG, et al. Supervised exercise versus primary stenting for claudication resulting from aortoiliac peripheral artery disease: six-month outcomes from the claudication: exercise versus endoluminal revascularization (clever) study. Circulation 2012;125:130-9.

24 Fakhry F, Spronk S, van der Laan L, et al. Endovascular revascularization and supervised exercise for peripheral artery disease and intermittent claudication: a randomized clinical trial. JAMA 2015;314:1936-44.

25 Mills JL, Conte MS, Armstrong DG, et al. The Society for vascular surgery lower extremity threatened limb classification system: risk stratification based on wound, ischemia, and foot infection (WIfl). J Vasc Surg 2014;59:220-34

26 Popplewell MA, Davies $H$, Jarrett $H$, et al. Bypass versus angio plasty in severe ischaemia of the leg - 2 (BASIL-2) trial: study protocol for a randomised controlled trial. Trials 2016;17:1-9. 
27 Menard MT, Farber A. The BEST-CLI trial: a multidisciplinary effort to assess whether surgical or endovascular therapy is better for patients with critical limb ischemia. Semin Vasc Surg 2014;27:82-4.

28 Mustapha JA, Katzen BT, Neville RF, et al. Determinants of long-term outcomes and costs in the management of critical limb ischemia: a population-based cohort study. J Am Heart Assoc 2018;7:e009724.

29 Wang JC, Kim AH, Kashyap VS. Open surgical or endovascular revascularization for acute limb ischemia. J Vasc Surg 2016;63:270-8

30 Enezate TH, Omran J, Mahmud E, et al. Endovascular versus surgical treatment for acute limb ischemia: a systematic review and meta-analysis of clinical trials. Cardiovasc Diagn Ther 2017;7:264-71

*31 Katsanos K, Spiliopoulos S, Kitrou P, et al. Risk of death following application of paclitaxel-coated balloons and stents in the femoropopliteal artery of the leg: a systematic review and meta-analysis of randomized controlled trials. J Am Heart Assoc 2018; 7:e011245
32 Nordanstig J, James S, Andersson M, et al. Mortality with paclitaxel-coated devices in peripheral artery disease. $N$ Engl J Med 2020;383:2538-46.

*33 Aboyans V, Ricco J-B, Bartelink M-LEL, et al. 2017 ESC guidelines on the diagnosis and treatment of peripheral arterial diseases, in collaboration with the European Society for vascular surgery (ESVS). Eur Heart J 2018;39:763-816.

*34 Gerhard-Herman MD, Gornik HL, Barrett C, et al. 2016 AHA/ACC guideline on the management of patients with lower extremity peripheral artery disease: a report of the American College of Cardiology/American heart association Task force on clinical practice guidelines. J Am Coll Cardiol 2017;69:e71-126.

*35 Aboyans V, Björck M, Brodmann M, et al. Questions and Answers on Diagnosis and Management of Patients with Peripheral Arterial Diseases: A Companion Document of the 2017 ESC Guidelines for the Diagnosis and Treatment of Peripheral Arterial Diseases, in collaboration with the European Society for Vascular Surgery (ESVS). Eur J Vasc Endovasc Surg 2018;55:457-64. 